\title{
Magnetic fields and ionized gas in the local group irregular galaxies IC 10 and NGC 6822
}

\author{
K. T. Chyży ${ }^{1}$, J. Knapik ${ }^{1}$, D. J. Bomans ${ }^{2}$, U. Klein ${ }^{3}$, R. Beck ${ }^{4}$, M. Soida ${ }^{1}$, and M. Urbanik ${ }^{1}$ \\ 1 Astronomical Observatory, Jagiellonian University, ul. Orla 171, 30-244 Kraków, Poland \\ 2 Astronomisches Institut der Ruhr-Universität Bochum, Universitätsstr. 150, 44780 Bochum, Germany \\ 3 Radioastronomisches Institut der Universität Bonn, Germany \\ ${ }^{4}$ Max-Planck-Institut für Radioastronomie, Postfach 2024, 53010 Bonn, Germany
}

Received 28 February 2003 / Accepted 24 April 2003

\begin{abstract}
We performed a high-sensitivity search for galaxy-scale magnetic fields by radio polarimetry at $10.45 \mathrm{GHz}$ and $4.85 \mathrm{GHz}$ with the Effelsberg $100 \mathrm{~m}$ radio telescope, accompanied by $\mathrm{H} \alpha$ imaging, for the two Local Group irregular galaxies IC 10 and NGC 6822. Their star-forming bodies are small and rotate slowly. IC 10 is known to have a very high star-forming activity, resembling blue compact dwarfs, while NGC 6822 has a low overall star-formation level. Despite very different current star formation rates, our $\mathrm{H} \alpha$ imaging revealed a large web of diffuse $\mathrm{H} \alpha$ filaments and shells in both IC 10 and NGC 6822. Some of them extend far away from the galaxy's main body. The total power emission of both objects shows bright peaks either at the positions of optically strong star-forming clumps (IC 10) or individual H II regions or supernova remnants (NGC 6822). However, in both cases we detect a smoothly distributed, extended component. In IC 10 we found clear evidence for the presence of a diffuse, mostly random magnetic field of $\simeq 14 \mu \mathrm{G}$ strength, probably generated by a fluctuation dynamo. One of the $\mathrm{H} \alpha$-emitting filaments appears to be associated with enhanced magnetic fields. We also rediscuss the reddening of IC 10 and its implications for its distance. In the case of NGC 6822 we found only very weak evidence for nonthermal emission, except perhaps for some regions associated with local gas compression. We detect in both galaxies small spots of polarized emission, indicative of regular fields $(\simeq 3 \mu \mathrm{G})$, at least partly associated with local compressional phenomena.
\end{abstract}

Key words. polarization - galaxies: irregular - galaxies: magnetic fields, galaxies: individual: IC10, NGC 6822 radio continuum: galaxies

\section{Introduction}

Irregular galaxies are low-mass objects exhibiting a variety of rotational properties with a subclass of them rotating very slowly (rotational speeds $V_{\text {rot }} \leq 30 \mathrm{~km} \mathrm{~s}^{-1}$ ) and often chaotically (e.g. Lo et al. 1993). They constitute important laboratories for large-scale interactions of stars with the interstellar medium: the low gravitational potential and relatively small size increase the probability that superbubbles, forming close to star-forming regions, may break out of the galaxy (e.g. Mac Low \& Ferrara 1998). Many irregular galaxies exhibit giant arcs or filaments of ionized gas (e.g. Sabbadin \& Bianchini 1979; Hunter et al. 1993; Bomans et al. 1997). The role of magnetic fields in the origin and confinement of these ionized structures (e.g. Hunter \& Gallagher 1990) is still a matter of debate.

In spiral galaxies magnetic fields, which are sufficiently strong to trigger star formation via magnetic instabilities (Blitz \& Shu 1980) or to influence the superbubble expansion (Ferriere et al. 1991), are probably generated by the mean-field dynamo (see Beck et al. 1996). This requires strong Coriolis

Send offprint requests to: K. Chyży, e-mail: chris@oa.uj.edu.pl forces (hence a rapid rotation) to give the turbulent motions a preferred sense of twisting. A sufficient size of the ionized gas envelope is also required. Large irregulars with star-forming bodies of $8-10 \mathrm{kpc}$ in diameter still posses significant regular fields (LMC - Klein et al. 1993, NGC 4449 - Chyży et al. 2000). They are explicable by non-standard dynamos driven by Parker instability (Moss et al. 1999; Hanasz \& Lesch 2000), working at low rotation speeds. The efficiency of postulated mechanisms is proportional to the ionized gas scale height, which may make them inefficient in very small irregulars having the star-forming bodies of $1-3 \mathrm{kpc}$ in diameter. Moreover, the magnetic field escape by diffusion is several times faster in such small objects than in large irregulars. Thus, small irregular galaxies should lack global magnetic fields (Chyży 2002). No relevant observational information on that matter existed up to now.

Spiral galaxies show much more regular magnetic fields in the interarm space than in strongly star-forming spiral arms (e.g. Beck \& Hoernes 1996). Nothing is known whether such a difference also occurs between small very strongly and very weakly star-forming irregulars. For all above reasons we performed a sensitive search for extended, diffuse total power 
and polarized emission in two small irregulars: IC 10 and NGC 6822 reflecting two extremities of star-forming activity. The star-forming body of IC 10 has the diameter of only $1.6 \mathrm{kpc}$ assuming the distance of $0.8 \mathrm{Mpc}$ (Wilson et al. 1996). Within this radius it rotates slowly at the speed $V_{\text {rot }} \simeq 30 \mathrm{~km} \mathrm{~s}^{-1}$ (Wilcots \& Miller 1998) and shows two regions of a very high star-forming activity. The mean $\mathrm{H} \alpha$ surface brightness of this galaxy is 4 times higher than in NGC 4449, making IC 10 a rapidly star-forming object similar to young blue compact galaxies (BCG, Richer et al. 2001). The optical body of NGC 6822 is physically about twice larger than IC 10. It lacks prominent regions of recent star formation. Both galaxies posses similar HI mass content: $1.7 \times 10^{8} M_{\odot}$ in the case of IC 10 (Huchtmeier 1979) and $1.3 \times 10^{8} M_{\odot}$ for NGC 6822 (de Blok \& Walter 2000).

As a search for weak extended emission is particularly difficult for NGC 6822 because of its large angular size and low radio surface brightness which makes single-dish observations preferred. For the sake of comparison both objects were studied in a way providing the best compromise between sensitivity and resolution, ensuring a similar number of beams per galaxy size. This was done by deep mapping of NGC 6822 at $4.85 \mathrm{GHz}$ and of IC 10 at $10.45 \mathrm{GHz}$ using the $100-\mathrm{m}$ Effelsberg radio telescope.

To study the associations of magnetic fields with the ionized gas and star formation we obtained sensitive images of both objects in the $\mathrm{H} \alpha$ line. The H II region content of IC 10 was analyzed by Hodge \& Lee (1990) and NGC 6822 by Hodge et al. (1988) but in the latter case only part of the galaxy was observed. Hunter et al. (1993) found that IC 10 is embedded in an impressive web of large filaments and shells, while they could not detect similar structures in NGC 6822. Our new $\mathrm{H} \alpha$ data cover each galaxy on one CCD frame with arcsecond resolution and high sensitivity. In Sect. 2 we present the observing techniques used to obtain the maps of total power, polarized intensity and $\mathrm{H} \alpha$ emission. Section 3. presents the observational results. The relative content of thermal and nonthermal emission and the question of global magnetic fields is discussed in Sect. 4.

\section{Observations and data reduction}

\subsection{Radio observations}

The total power and polarization maps of IC 10 at $10.45 \mathrm{GHz}$ were obtained using the four-horn system in the secondary focus of the Effelsberg 100-m MPIfR telescope (Schmidt et al. $1993)^{1}$, the details of the observing procedure were described in detail in our previous works (e.g. Soida et al. 1996). In total we obtained 28 coverages of IC 10 scanned in the azimuthelevation frame. NGC 6822 was observed in total power and polarization at $4.85 \mathrm{GHz}$ using the two-horn system in the secondary focus of the Effelsberg telescope. We obtained 9 azimuth-elevation coverages of this galaxy.

\footnotetext{
1 The $100-\mathrm{m}$ telescope at Effelsberg is operated by the Max-Planck-Institut für Radioastronomie (MPIfR) on behalf of the Max-Planck-Gesellschaft.
}

The telescope pointing was checked at time intervals of about 2 hours by making cross-scans of nearby strong point sources. The flux density scale was calibrated by mapping the highly polarized source 3C286. Its total power flux density of $4.47 \mathrm{Jy}$ at $10.45 \mathrm{GHz}$ and $7.44 \mathrm{Jy}$ at $4.85 \mathrm{GHz}$ has been adopted using the formulae by Baars et al. (1977). The same calibration factors were used for total power and polarized intensity, yielding a mean degree of polarization of $3 \mathrm{C} 286$ of $12.2 \%$ at $10.45 \mathrm{GHz}$ and $10.5 \%$ at $4.85 \mathrm{GHz}$, in reasonable agreement with other published values (Tabara \& Inoue 1980).

The data reduction was performed using the NOD2 data reduction package (Haslam 1974). By combining the information from appropriate horns, using the "software beam-switching" technique (Morsi \& Reich 1986) followed by a restoration of total intensities (Emerson et al. 1979), for each coverage we obtained I, Q and U maps. These were combined into final maps of total power, polarized intensity, polarization degree and polarization position angles using the spatial frequency weighting method (Emerson \& Gräve 1988). A digital filtering process, which removes spatial frequencies corresponding to noisy structures smaller than the telescope beam, was applied to the final maps.

The original beam of our $10.45 \mathrm{GHz}$ observations was 1'13, corresponding to $270 \mathrm{pc}$ at the distance to IC 10 of $820 \mathrm{kpc}$ (Wilson et al. 1996). At the distance to NGC 6822 of $490 \mathrm{kpc}$ (McGonegal et al. 1983) the original beam of 2.5 at $4.85 \mathrm{GHz}$ corresponds to $360 \mathrm{pc}$. To increase the sensitivity to extended structures we also used data convolved to 1:3 (IC 10) and $3^{\prime}$ (NGC 6822).

\subsection{Observations in $\mathrm{H} \alpha$ line}

\subsubsection{IC 10}

We observed IC 10 using the Calar Alto Observatory $1.23 \mathrm{~m}$ telescope in October 1994. A Tektronics $1024^{2}$ pixel CCD was used as the detector resulting in a spatial scale of $00^{\prime} 502$ per pixel. The seeing during our observations was 1"3 3 and the conditions were non-photometric, with light cirrus suspected. We took two 30 min exposures through a $75 \AA$ wide filter centered at $6560 \AA$, well matched to the $\mathrm{H} \alpha$ line of IC 10 $\left(V_{\mathrm{rad}}=-360 \mathrm{~km} \mathrm{~s}^{-1}\right)$. For the continuum image we observed IC 10 with a standard Johnson-Cousins $R$ filter $(10 \mathrm{~min})$. The data reduction was performed in the standard way using the IRAF package. We applied small kernal Gaussian filters to both the $\mathrm{H} \alpha$ and the $R$ image to match the slightly different point spread functions. After this step the continuum subtraction was performed, as described in e.g. Bomans et al. (1997). Due to the poor photometric conditions we performed the flux calibration relative to the $\mathrm{H} \alpha$ maps of Hodge \& Lee (1990). The major uncertainty of this process is the choice of the aperture and centering. The scatter of the correlation implies that our calibration has an uncertainty of $25 \%$. The correction of our $\mathrm{H} \alpha$ fluxes for the contribution of [N II] is less than 1.2 for $\mathrm{H}$ II regions. The contribution may be higher for the filaments, due to the larger $[\mathrm{N} \mathrm{II}] / \mathrm{H} \alpha$ ratio in the diffuse ionized gas (DIG) (see e.g. Dettmar 1993). 


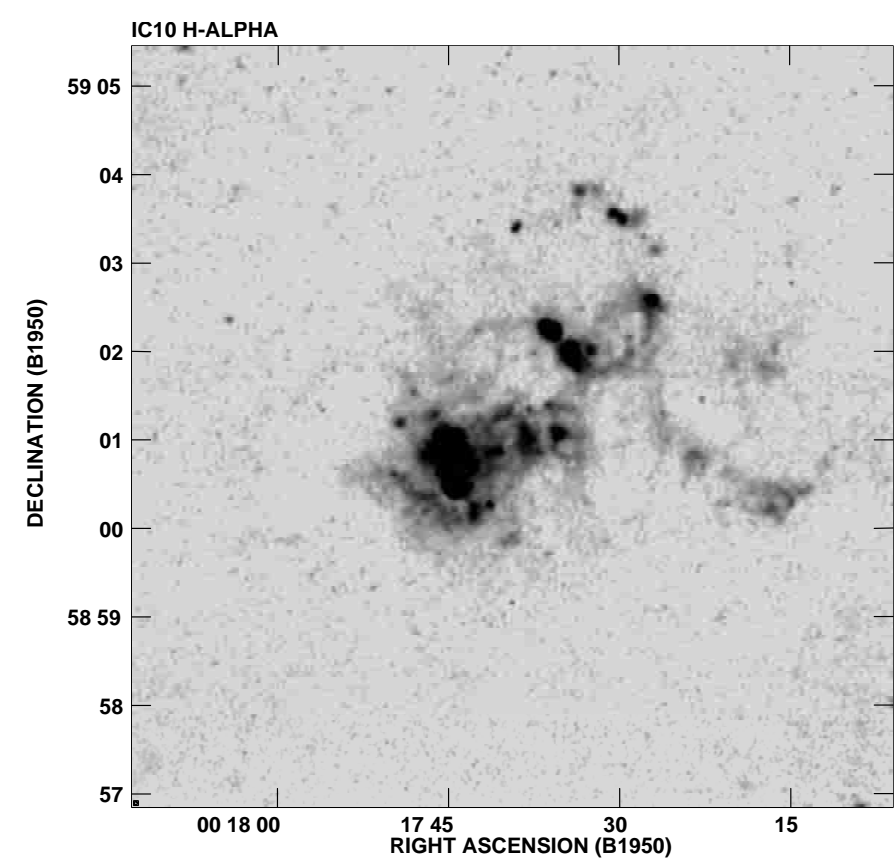

Fig. 1. H $\alpha$ image of IC 10, free from the stellar continuum.

\subsubsection{NGC 6822}

The optical data for NGC 6822 were taken with the $1 \mathrm{~m}$ telescope at Mt. Laguna Observatory using a Loral/lesser $2048^{2}$ pixel CCD giving a scale of $0.41^{\prime \prime} /$ pix. The data reduction techniques were the same as for the observations of IC 10. Seeing was around 1'.5. We observed NGC 6822 twice for $1800 \mathrm{~s}$ in an $\mathrm{H} \alpha$ filter and for $600 \mathrm{~s}$ in a broad band $R$ filter. The photometric conditions during observation of NGC 6822 were unstable during the run. We therefore decided to calibrate our data relatively to the published $\mathrm{H}$ II region fluxes by Hodge $\&$ Lee (1990). The quality of the correlation is similar to that for IC 10 , as is also the correction of the flux scale for contribution of [N II].

\section{Results}

\subsection{Ho image of IC 10}

Our $\mathrm{H} \alpha$ image of IC 10 (Fig. 1) shows the H II region complexes in the SE and NW of the galaxy as well as the extended diffuse ionized medium of the galaxy. Many of the features were already described by Hunter et al. (1993). We point to a few features important for further discussion: most of the diffuse emission is actually in filaments and shells, there is no evidence for an unresolved component. The filaments and shells extend out to $0.6 \mathrm{kpc}$ from the main body of IC 10, and not all of them originate in the two dominant star forming regions. Two very prominent $\mathrm{H} \alpha$-emitting features extend westwards from the northern star-forming region. We also detect a faint bubble-shaped $\mathrm{H} \alpha$ feature SW of the prominent dust lane in the southern part of IC 10, coincident with the position of the non-thermal superbubble detected there by Yang \& Skillman (1993).

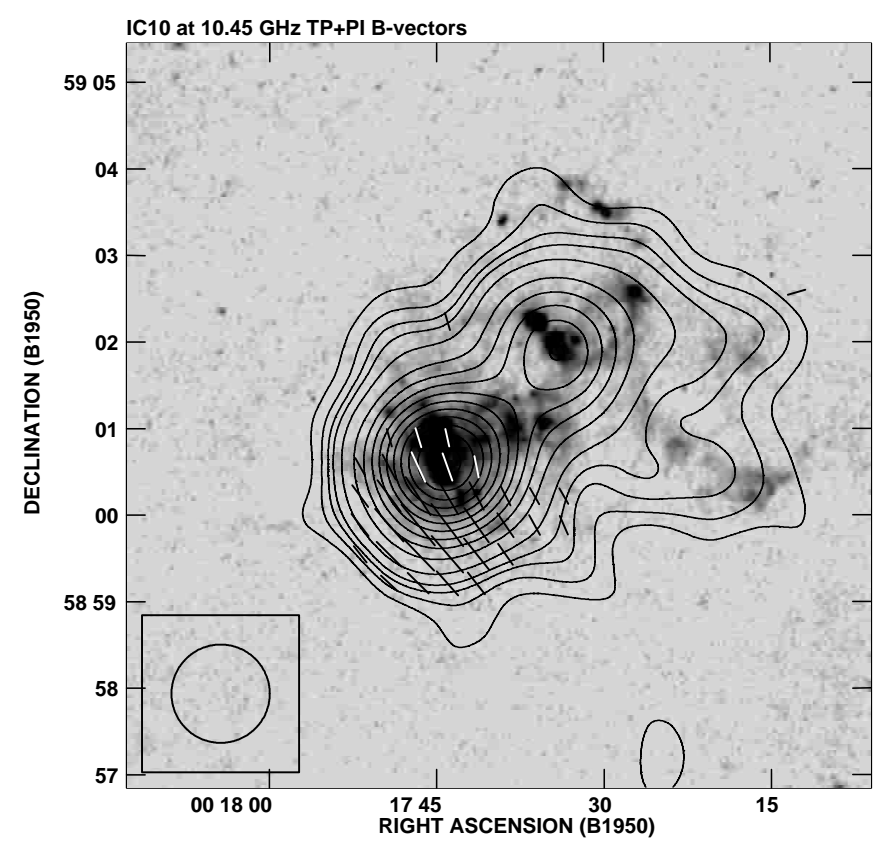

Fig. 2. Total power contour map of IC 10 at $10.45 \mathrm{GHz}$, with $\boldsymbol{B}$-vectors of polarized intensity superimposed onto the $\mathrm{H} \alpha$ image. The resolution is 1 '.13. Contour levels are 2, 3, 4, 5 then 7, 11, 15 etc. $\mathrm{mJy} / \mathrm{b}$.a., the vector of $1^{\prime}$ length corresponds to polarized intensity of $1.5 \mathrm{mJy} / \mathrm{b}$.a.

\subsection{Radio continuum of IC 10}

The $10.45 \mathrm{GHz}$ total power map of IC 10 is shown in Fig. 2 at full resolution with superimposed $\boldsymbol{B}$-vectors of polarized intensity. The map has an rms noise of $0.6 \mathrm{mJy} / \mathrm{b}$.a. Two bright, poorly resolved total power peaks are found at the positions of optically bright star-forming regions as already stated by Klein et al. (1983). They are present in Condon's (1987) map at $1.49 \mathrm{GHz}$, thus contain some nonthermal component, in agreement with earlier findings by Klein \& Gräve (1986). In addition IC 10 shows smooth, extended emission (visible in Condon's map, too) extending westwards up to $0.7 \mathrm{kpc}$ from the star formation nests. In the western disk, the diffuse total power emission makes two extensions coincident with the $\mathrm{H} \alpha$ emitting filaments mentioned in the previous section. This region coincides also with a faint, diffuse optical glow. Integration of the total power map in elliptical rings with an inclination of $33.6^{\circ}$ and position angle of $136^{\circ}$, both taken from the Lyon-Meudon Extragalactic Database (LEDA), yields an integrated flux density of IC 10 at $10.45 \mathrm{GHz}$ of $155 \pm 16 \mathrm{mJy}$.

Our polarized intensity map of IC 10 (Fig. 3) has an rms noise of $0.14 \mathrm{mJy} / \mathrm{b}$.a. The polarized brightness shows a single, weakly resolved peak south of a straight, linear dust feature crossing the outskirts of the southern star-forming region. The degree of polarization at the polarized intensity peak is about $4 \%$ increasing to $8-10 \%$ at its southern boundary. The orientation of the $\boldsymbol{B}$-vectors is parallel to the dust lane. IC 10 lies close to the galactic plane, where the Faraday rotation measures may reach $200 \mathrm{rad} / \mathrm{m}^{2}$ (Simard-Normandin \& Kronberg 1980). At the frequency of $10.45 \mathrm{GHz}$ this corresponds to a polarization angle offset by $9^{\circ}$, which does not affect strongly the 


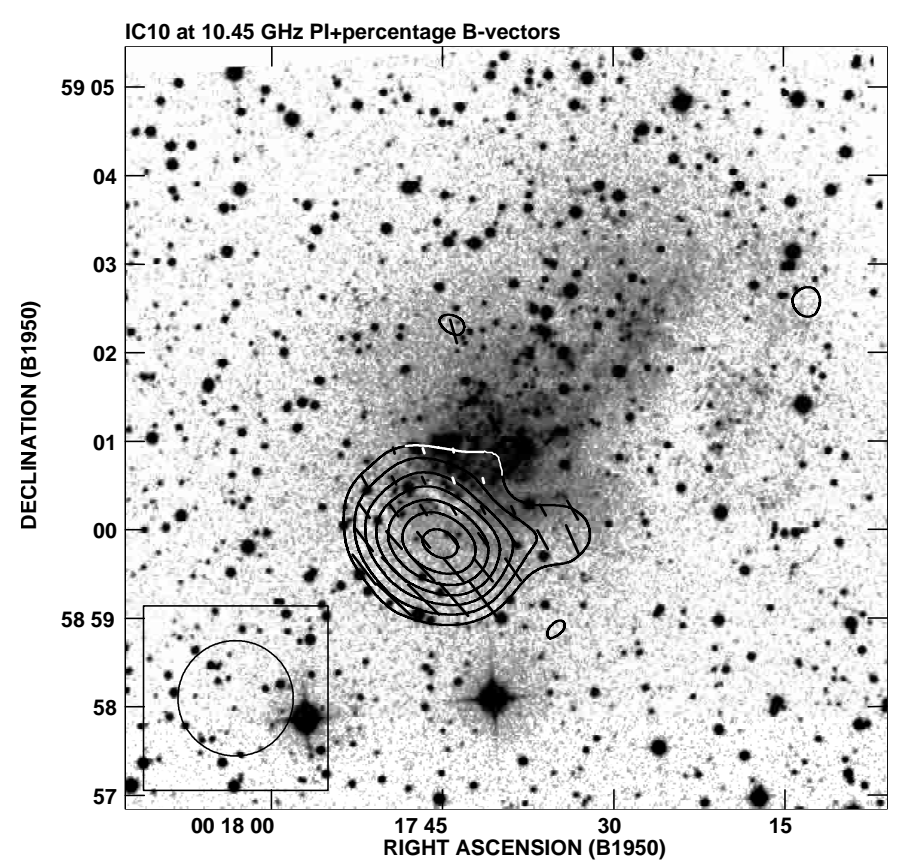

Fig. 3. Contour map of the polarized intensity of IC 10 at $10.45 \mathrm{GHz}$, with $\boldsymbol{B}$-vectors of the polarization degree superimposed onto an optical image from DSS. The resolution is 1 .'3. The contour levels are 0.3 , 0.4 etc. $\mathrm{mJy} / \mathrm{b} . \mathrm{a}$., the vector of $1^{\prime}$ length corresponds to polarization degree of $15 \%$.

alignment with the dust lane. The bright total power peaks are found to be completely unpolarized, with polarization degrees at their position smaller than $0.7 \%$. The diffuse emission in the northern disk and the western extension are polarized by less than $0.8 \%$.

The polarized feature is not associated with any discrete sources visible in Condon's (1987) map and is unlikely to be due to a background source. Instead, it coincides with a diffuse nonthermal spur found there by Yang \& Skillman (1993). Integration of the polarized intensity map shown in Fig. 3 in the same rings as the total power one yields an integrated polarized flux density of IC 10 at $10.45 \mathrm{GHz}$ of $1.9 \pm 1.1 \mathrm{mJy}$. This implies a mean polarization degree of $1.2 \pm 0.7 \%$, much lower than in normal spirals (Knapik et al. 2000). All the detected polarized flux comes from the barely resolved blob in the southern region. After its subtraction as an unresolved source, the polarization degree drops below $0.5 \%$.

\section{3. $H \alpha$ image of NGC 6822}

Our new continuum-free $\mathrm{H} \alpha$ image of NGC 6822 (Fig. 4) covers only the central part of the galaxy. Its detailed discussion will be presented by Bomans (in prep.). It shows small H II regions at several positions in the galaxy's body. Some H II regions beyond the radius of $1 \mathrm{kpc}$ are seen for the first time. The size and luminosity of the H II regions outside the main galaxy's body are larger than those inside it. No large, dominant star-forming complexes like those in IC 10 were found. Giant H II regions are missing in NGC 6822, too.

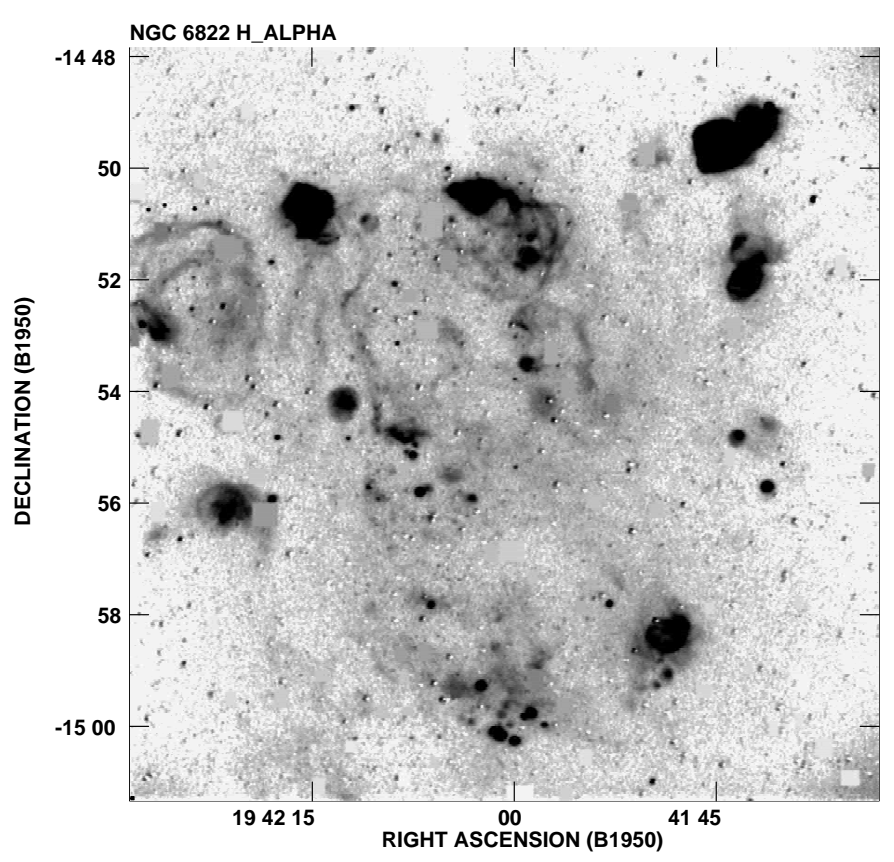

Fig. 4. $\mathrm{H} \alpha$ image of NGC 6822, free from the contribution from the stellar continuum.

Our data confirm the large difference in current star formation between IC 10 and NGC 6822, the former one having more H II regions and they are more luminous than in NGC 6822. The star-forming regions of NGC 6822 are distributed over a much larger surface area than those in IC 10. The number of superbubbles is also larger in NGC 6822 than in IC 10. In the latter the $\mathrm{H}$ II region population is dominated by compact H II regions, occasionally accompanied by high surface brightness arcs.

Surprisingly, despite its very low current star formation rate NGC 6822 shows an extended network of diffuse $\mathrm{H} \alpha$ filaments and shells, seen for the first time in the present work. They are filling almost the whole optically bright galaxy. Some of them extend out to $1.5 \mathrm{kpc}$ from the main body, especially to the NE.

\subsection{Radio continuum of NGC 6822}

Our total power map of NGC 6822 at $4.85 \mathrm{GHz}$ (Fig. 5) has an $\mathrm{rms}$ noise of $0.65 \mathrm{mJy} / \mathrm{b} . \mathrm{a}$. Some individual point sources coincide with isolated H II regions and supernova remnants. However, the brightest peak east of the optically bright galaxy and the double source in its southern part have no obvious optical counterparts. In addition to these discrete sources, seen also in the $1.49 \mathrm{GHz}$ map by Condon (1987), we detect diffuse emission extending westward from the brightest peak over the optically bright body and filling the gaps between individual sources. The diffuse radio emission west of the strongest radio peak was also marginally detected by Klein \& Gräve (1996), while in our map we could measure it at a level of at least $5 \sigma$ rms noise (see Sect. 4). Integration of the total power map in elliptical rings with an inclination of $25^{\circ}$ and a position angle of $5^{\circ}$ (both taken from the LEDA database) yield an integrated flux density of NGC 6822 at $4.85 \mathrm{GHz}$ of $147 \pm 19 \mathrm{mJy}$. 


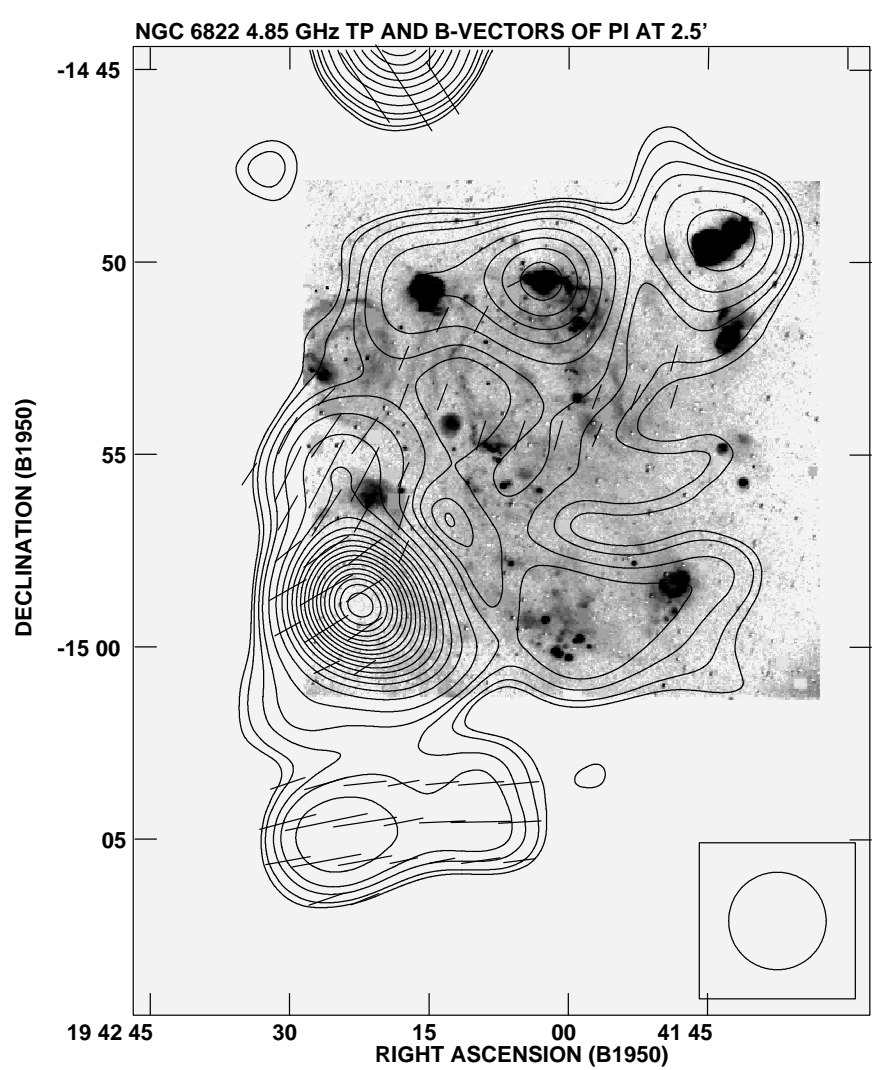

Fig. 5. Total power contour map of NGC 6822 at $4.85 \mathrm{GHz}$ with $\boldsymbol{B}$-vectors of polarized intensity superimposed onto a $\mathrm{H} \alpha$ image. The resolution is 2.5 . Contour levels are $1.5,2,3,4$, then $6,9,12$ etc. $\mathrm{mJy} / \mathrm{b}$.a., the vector of $1^{\prime}$ length corresponds to polarized intensity of $0.5 \mathrm{mJy} / \mathrm{b} . \mathrm{a}$.

The background-like strong source located at $\mathrm{RA}_{1950}=$ $19^{\mathrm{h}} 42^{\mathrm{m}} 22^{\mathrm{s}} .9 \mathrm{Dec}_{1950}=-14^{\circ} 58^{\prime} 43^{\prime \prime}$ with a flux of $48 \mathrm{mJy}$ (see Sect. 4.1.3) was subtracted in this derivation.

Our map of the polarized intensity of NGC 6822 at $4.85 \mathrm{GHz}$ (Fig. 6) has an rms noise of $0.1 \mathrm{mJy} / \mathrm{b}$.a. The bright total power source east of the optical galaxy (a background object?) is weakly polarized, with a degree of polarization of about $2 \%$. Significant polarization was detected to extend northwards of it, with a degree of polarization of about $8 \%$. Significant polarization (mean degree of about $13 \%$ ) was detected in the double structure (also background sources?) in the southern galaxy's outskirts. Integration of the map in the same elliptical rings as the total power one yields an integrated polarized flux density of NGC 6822 at $4.85 \mathrm{GHz}$ of $5.1 \pm 2.1 \mathrm{mJy}$ implying a mean polarization degree at this frequency of $3.5 \pm 1.5 \%$, most of it being due to the mentioned discrete sources. However, the diffuse radio emission filling the inner galaxy (excluding possible background sources and those related to star-forming regions in the north) shows a mean polarization degree of $5 \pm 1 \%$. The degree of diffuse polarization is highest in the rectangular region delineated by $\mathrm{RA}_{1950}=19^{\mathrm{h}} 41^{\mathrm{m}} 40^{\mathrm{s}}$ and $19^{\mathrm{h}} 42^{\mathrm{m}} 13^{\mathrm{s}}, \operatorname{Dec}_{1950}=-14^{\circ} 57^{\prime}$ and $-14^{\circ} 52^{\prime}$ and reaches $15 \pm 2 \%$, extending over an area of about 6 beam areas.

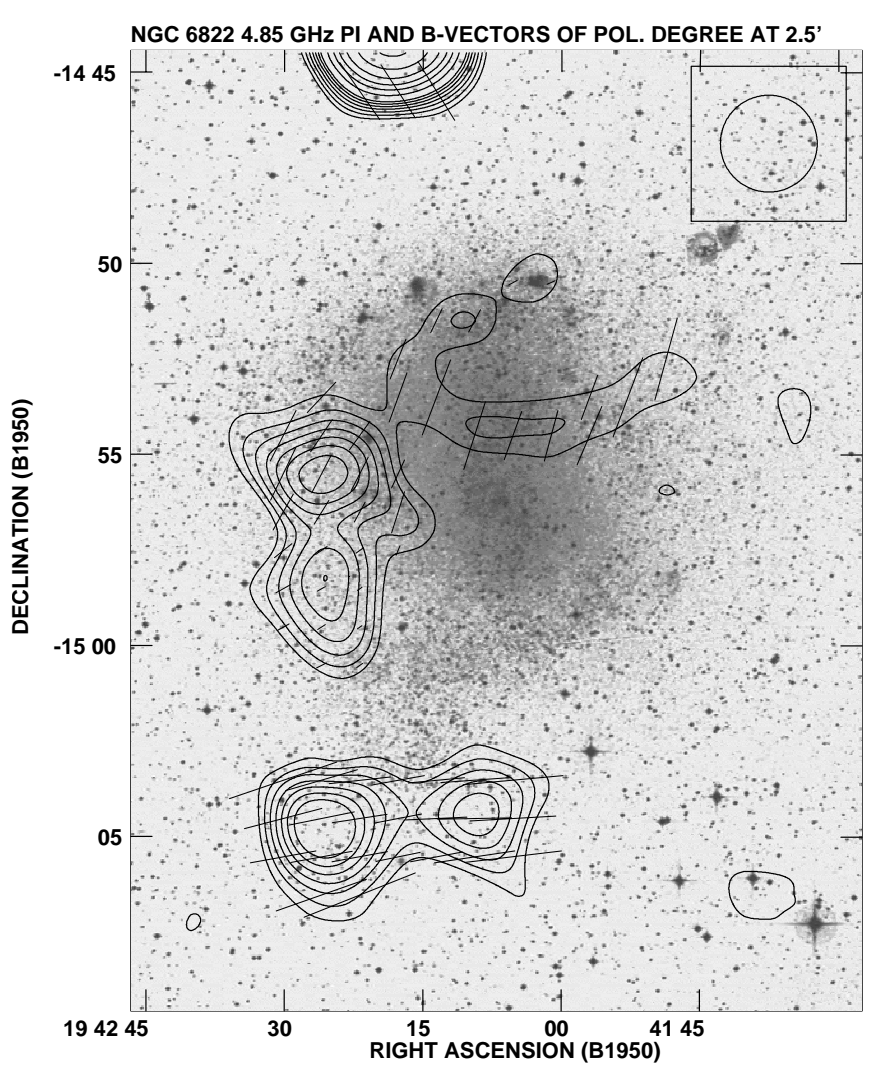

Fig. 6. Contour map of the polarized intensity of NGC 6822 at $4.85 \mathrm{GHz}$ with $\boldsymbol{B}$-vectors of the polarization degree superimposed onto an optical image from DSS. The resolution is 2'.5. Contour levels are $0.3,0.4,0.5$ etc. $\mathrm{mJy} / \mathrm{b} . \mathrm{a}$., the vector of $1^{\prime}$ length corresponds to polarization degree of $8 \%$.

\section{Discussion}

\subsection{Thermal and nonthermal emission}

\subsubsection{Thermal emission from the rapidly star-forming IC 10}

The radio spectrum of IC 10 between $1.4 \mathrm{GHz}$ and $24 \mathrm{GHz}$ is presented in Fig. 7. The mean spectral index is $\alpha=0.33 \pm 0.02$ $\left(S_{v} \propto v^{-\alpha}\right)$. This is much less than the source spectrum of cosmic-ray (CR) electrons accelerated in shocks, yielding $\alpha=0.5$. The spectrum observed in normal galaxies, involving synchrotron losses and energy-dependent diffusion (Niklas et al. 1997) is even steeper. Our attempts to model a combination of thermal and nonthermal components (the latter with an adjustable slope) yields the combination of a nonthermal spectral index $\alpha_{\mathrm{nt}}=0.55 \pm 0.05$ and a mean thermal fraction $\overline{f_{\mathrm{th}}}=0.6 \pm 0.1$ as the best reproduction of the data (Fig. 7). Nevertheless, higher $\overline{f_{\text {th }}}$ reaching $0.7-0.75$ corresponding to $\alpha_{\mathrm{nt}} \simeq 0.66-0.74$ cannot be excluded on the basis of radio spectrum alone.

We note that the radio spectrum of IC 10 is considerably flatter than that of the much larger irregular NGC 4449, the latter having an observed spectral slope of $0.41 \pm 0.01$ (Klein et al. 1996). These authors determined $\alpha_{\mathrm{nt}}=0.7 \pm 0.14$ and $\overline{f_{\mathrm{th}}}$ (converted to $10.45 \mathrm{GHz}$ ) of 0.4. The large irregular NGC 4449 seems to be an intermediate case between large spirals and the 


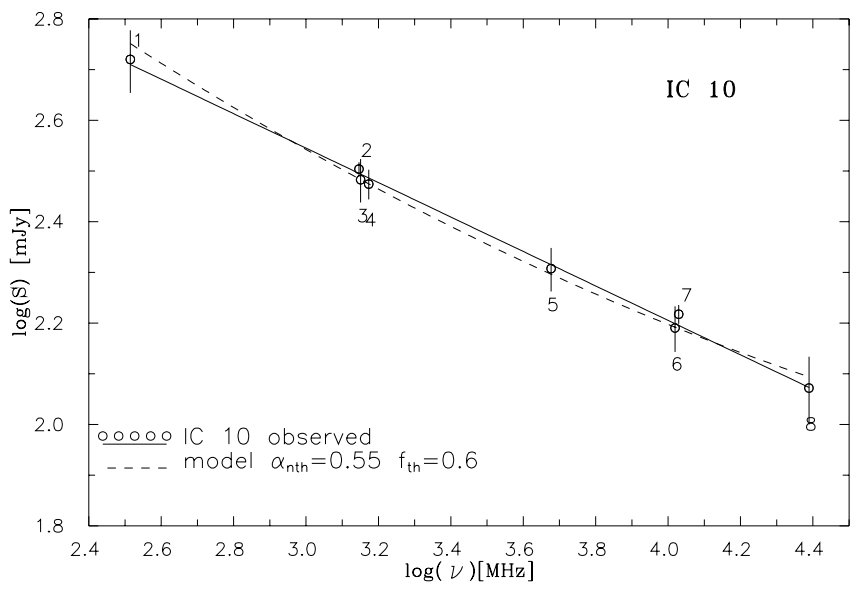

Fig. 7. The radio spectrum of IC 10. The numbers label the data sources: 1. Computed by us from the WENSS survey maps (Rengelink et al. 1997), 2. White \& Becker (1992), 3. Shostak (1974), 4. Condon (1987), 5. Klein et al. (1983), 6. this work, 7. Klein et al. (1983), 8. Klein \& Gräve (1986). The dashed line shows the best model spectrum resulting from a combination of maximum thermal fraction of $60 \%$ and a nonthermal spectral index of 0.55 .

small irregular galaxy IC 10 concerning both thermal fraction and nonthermal spectrum slope.

Being aware of uncertainties due to extinction we verified our estimates of the thermal flux using the $\mathrm{H} \alpha$ map convolved to the resolution of $72^{\prime \prime}$. To determine the extinction we compared the radio fluxes of thermal discrete sources in the $6 \mathrm{~cm}$ map of Yang \& Skillman (1993) to the predictions from the $\mathrm{H} \alpha$ emission of associated $\mathrm{H}$ II regions. As a mean value we obtained $A_{\mathrm{H} \alpha}=4.35$. We note however, that the metallicity of IC 10 is several times smaller than that of our Galaxy (Lequeux et al. 1979), thus most of extinction originates in the Galactic foreground, especially at the Galactic latitude of $-3.3^{\circ}$ (LEDA database). As small variations of Galactic extinction over the angular size of IC 10 is expected this value has been applied to the whole map. The results were compared to those obtained from the radio spectrum. The difference at $10.45 \mathrm{GHz}$ reaches $6 \mathrm{mJy} / \mathrm{b} . \mathrm{a}$. in the northern blob and $4 \mathrm{mJy} / \mathrm{b}$.a. in the southern one, thus on average it constitutes less than $30 \%$ of the thermal brightness. This is the maximum uncertainty of all assumptions used in determining the thermal flux (including constant extinction across the galaxy). In the outer disk we find good agreement between the thermal brightness estimated from the radio spectrum and the $\mathrm{H} \alpha$ emission. As the determination of the thermal flux from the $\mathrm{H} \alpha$ brightness was independent of our assumption concerning $\alpha_{\mathrm{nt}}$, this supports in retrospect our adopted values of $\alpha_{\mathrm{nt}}=0.55$ and $\overline{f_{\mathrm{th}}}=0.6$.

Our estimate of the extinction results in $E_{(B-V)}=1.74$, assuming a Mathis (1979) reddening curve. This is very similar to what was derived by Yang \& Skillman (1993) using the same method, but a magnitude higher than the value obtained from an analysis of the colour-magnitude diagram (CMD) of IC10, $E_{(B-V)}=0.77$ (Hunter 2000; Massey \& Armandroff 1995). Since a global high value of the reddening seems incompatible with the CMD analysis (Hunter 2000), we are forced to propose that the $\mathrm{H}$ II regions show significant internal reddening, and/or

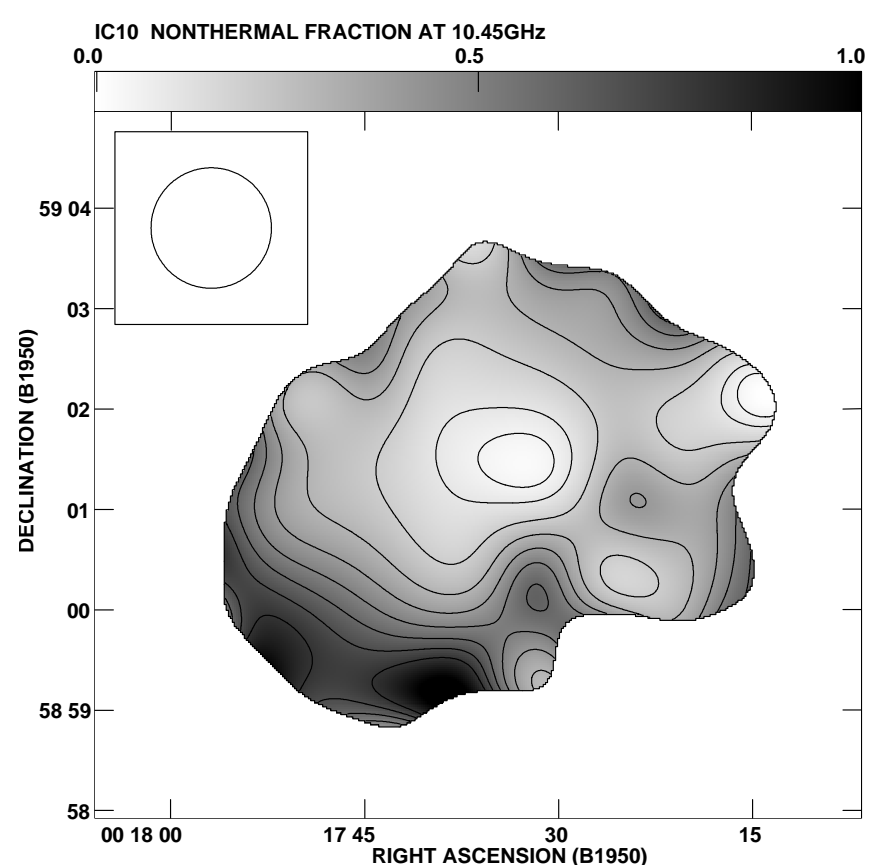

Fig. 8. Distribution of the nonthermal fraction in IC 10 computed from the spectral index map between $1.49 \mathrm{GHz}$ (Condon 1987) and our map shown in Fig. 2, assuming a nonthermal spectral index of 0.55. The point sources listed in Table 1 were subtracted.

there are pockets of high foreground reddening. The first possibility is unlikely, taking into account a reasonable agreement between our thermal fluxes derived from the radio spectrum (insensitive to extinction) and the $\mathrm{H} \alpha$ line, assuming the same extinction in H II regions and diffuse gas. Still, it is interesting to note here, that the foreground extinction maps of Schlegel et al. (1998) give a value for the foreground reddening alone which is very similar to our value. Clearly the debate about the reddening of IC10 is still not settled yet. Since the reddening has implications for the distance and the interpretation of the fluxes new approaches could really help here. It is important to use the Balmer decrement method for H II regions and the widespread diffuse emission to derive a fine gridded reddening map of IC 10.

\subsubsection{Nonthermal emission from IC 10}

Using the spectral index distribution computed between our and Condon's (1987) map at $1.49 \mathrm{GHz}$ and assuming a constant nonthermal spectral index of 0.55 over the whole galaxy, we have obtained the distribution of thermal and nonthermal fractions in IC 10 at $10.45 \mathrm{GHz}$ (the latter shown in Fig. 8). We computed the $10.45 \mathrm{GHz}$ fluxes of point sources present in the maps of Yang \& Skillman (1993, Table 1) and subtracted them from our total power map, thus the figure refers to diffuse emission only. The nonthermal fraction shows a minimum at the positions of the north-western bright star-forming region, dropping to zero there independently of the assumed $\alpha_{\mathrm{nt}}$. The nonthermal emission associated with the southern star-forming region is larger and amounts to $35 \%$. If a steeper nonthermal spectrum is assumed, e.g. $\alpha_{\mathrm{nt}}=0.7$ this value changes 
Table 1. Point sources subtracted from IC 10 maps.

\begin{tabular}{ccrr}
\hline \hline $\mathrm{RA}_{1950}$ & Dec $_{1950}$ & $\begin{array}{r}S_{1.49} \\
{[\mathrm{mJy}]}\end{array}$ & $\begin{array}{r}S_{10.45} \\
{[\mathrm{mJy}]}\end{array}$ \\
\hline $00^{\mathrm{h}} 17^{\mathrm{m}} 44^{\mathrm{s}} .1$ & $59^{\circ} 00^{\prime} 28^{\prime \prime}$ & 3.6 & 3.0 \\
$00^{\mathrm{h}} 17^{\mathrm{m}} 44^{\mathrm{s}} .9$ & $59^{\circ} 00^{\prime} 31^{\prime \prime}$ & 2.2 & 1.7 \\
$00^{\mathrm{h}} 17^{\mathrm{m}} 43.9$ & $59^{\circ} 00^{\prime} 51^{\prime \prime}$ & 3.8 & 4.2 \\
$00^{\mathrm{h}} 17^{\mathrm{m}} 44.6$ & $59^{\circ} 01^{\prime} 01^{\prime \prime}$ & 2.6 & 1.8 \\
$00^{\mathrm{h}} 17^{\mathrm{m}} 34.4$ & $59^{\circ} 02^{\prime} 02^{\prime \prime}$ & 10.4 & 8.4 \\
$00^{\mathrm{h}} 17^{\mathrm{m}} 36^{\mathrm{s}} .2$ & $59^{\circ} 02^{\prime} 17^{\prime \prime}$ & 2.0 & 1.3 \\
$00^{\mathrm{h}} 17^{\mathrm{m}} 31^{\mathrm{s}} .9$ & $59^{\circ} 02^{\prime} 16^{\prime \prime}$ & 2.4 & 0.6 \\
\hline
\end{tabular}

to $20 \%$. The nonthermal fraction rises gradually towards the southern galaxy boundary, reaching some $70 \%-90 \%(\simeq 45 \%-$ $55 \%$ for $\alpha_{\mathrm{nt}}$ of 0.7 ) in the nonthermal blob observed by Yang \& Skillman (1993). We state the existence of significant magnetic fields (partly regular) in this region. Very little nonthermal emission is observed from the northern $\mathrm{H} \alpha$-emitting clump on the western galaxy side, where the total power extension is almost purely thermal. A long filament extending from IC 10 to the SW is accompanied by a ridge with a nonthermal fraction of about $40 \%$ (assuming $\alpha_{\mathrm{nt}}=0.55$ ).

In IC 10, which is forming stars more intensively than NGC 6822 we detected unambiguously a nonthermal component of its radio continuum emission (amounting to 30\%-40\%) of the total flux, indicating a widespread magnetic field. Its mean equipartition strength within the area delineated by $5 \%$ of the maximum brightness (after the subtraction of sources from Yang \& Skillmann 1993, Table 1) is $14 \pm 4 \mu \mathrm{G}$, changing only little whether we assume either a thermal fraction of $60 \%$ and a nonthermal spectral index of 0.55 or $70 \%$ thermal fraction and a nonthermal spectral index of 0.65 . We adopted a lower energy cutoff of $300 \mathrm{MeV}$, a proton-to electron ratio of 100 (Pacholczyk 1970) and a disk thickness of $0.8 \mathrm{kpc}$ (assuming that IC 10 is roughly a prolate ellipsoid). The error of the magnetic field strength includes the uncertainty of these quantities by a factor of 2 . The minimum field strength obtained by varying all assumptions is $10 \mu \mathrm{G}$. In the disk outskirts we obtain a total equipartition magnetic field of $7 \mu \mathrm{G}$. These values are comparable to total equipartition fields in massive and strongly star-forming spirals. Apparently the small size of IC 10 does not prevent it from developing strong total (mostly random) magnetic field. The nonthermal emission and the total magnetic field thus fills the entire body of IC 10, dominating in its southern region.

The best agreement between the thermal emission of IC 10 determined from the radio spectrum and $\mathrm{H} \alpha$ emission implies $\alpha_{\mathrm{nt}}=0.55$, hence a CR electron energy spectrum even harder than in NGC 4449 (Klein et al. 1996). In fact, apart from the western extension the total power emission drops almost to zero at a distance of $\simeq 400-500 \mathrm{pc}$ from the large star-forming complexes, compared to a typical scale length of $\simeq 1 \mathrm{kpc}$ in normal spirals. This may mean the lack of an extended magnetic halo and no space where the electrons could lose efficiently their energy. The lack of an extended halo also allows fast escape of cosmic rays - they seem to be only weakly confined by the magnetic field. The same conclusion has been reached by
Table 2. Spectral index of discrete sources subtracted from the map of NGC 6822.

\begin{tabular}{llrr}
\hline \hline $\mathrm{RA}_{1950}$ & Dec $_{1950}$ & $\begin{array}{r}S_{4.85} \\
\mathrm{mJy}\end{array}$ & $\begin{array}{r}\text { spectral } \\
\text { index }\end{array}$ \\
\hline $19^{\mathrm{h}} 41^{\mathrm{m}} 51^{\mathrm{s}} .1$ & $-14^{\circ} 48^{\prime} 00^{\prime \prime}$ & $3.0 \pm 0.7$ & $0.86 \pm 0.23$ \\
$19^{\mathrm{h}} 41^{\mathrm{m}} 43^{\mathrm{s}} .3$ & $-14^{\circ} 49^{\prime} 27^{\prime \prime}$ & $9.8 \pm 0.8$ & $0.14 \pm 0.10$ \\
$19^{\mathrm{h}} 42^{\mathrm{m}} 15^{\mathrm{s}} .5$ & $-14^{\circ} 50^{\prime} 38^{\prime \prime}$ & $7.4 \pm 0.8$ & $0.16 \pm 0.12$ \\
$19^{\mathrm{h}} 42^{\mathrm{m}} 02^{\mathrm{s}} .9$ & $-14^{\circ} 50^{\prime} 30^{\prime \prime}$ & $17.9 \pm 0.7$ & $0.11 \pm 0.06$ \\
$19^{\mathrm{h}} 42^{\mathrm{m}} 25^{\mathrm{s}} .0$ & $-14^{\circ} 55^{\prime} 36^{\prime \prime}$ & $11.2 \pm 0.8$ & $0.79 \pm 0.07$ \\
$19^{\mathrm{h}} 42^{\mathrm{m}} 22^{\mathrm{s}} .9$ & $-14^{\circ} 58^{\prime} 43^{\prime \prime}$ & $48.4 \pm 2.0$ & $0.96 \pm 0.04$ \\
$19^{\mathrm{h}} 42^{\mathrm{m}} 17^{s} .8$ & $-14^{\circ} 59^{\prime} 49^{\prime \prime}$ & $16.4 \pm 1.5$ & $0.57 \pm 0.08$ \\
$19^{\mathrm{h}} 42^{\mathrm{m}} 24^{\mathrm{s}} .9$ & $-15^{\circ} 04^{\prime} 51^{\prime \prime}$ & $8.2 \pm 1.0$ & $0.65 \pm 0.12$ \\
$19^{\mathrm{h}} 42^{\mathrm{m}} 09^{\varsigma} .3$ & $-15^{\circ} 04^{\prime} 24^{\prime \prime}$ & $4.2 \pm 0.7$ & $0.67 \pm 0.16$ \\
\hline
\end{tabular}

Klein et al. (1991) for a larger sample of BCG's, which show a clear radio spectrum steepening with increasing luminosity, thus also with the mass of the stellar body. The thermal content seems to increase and the spectrum of CR electrons gets harder (as they are weaker confined) in a continuous way from massive spirals, through large irregulars to small, compact rapidly star-forming galaxies.

\subsubsection{Any nonthermal emission from the weakly star-forming NGC 6822?}

The analysis of the radio spectrum of NGC 6822 is difficult because of the large angular extent and the too low level of emission. Attempts to construct a spectral index map between $4.85 \mathrm{GHz}$ and $1.49 \mathrm{GHz}$ led to unphysical results because of severe flux losses at the latter frequency, even in the vicinity of bright peaks. Instead, we attempted to determine the flux densities at $4.85 \mathrm{GHz}$ of particular point sources identifiable in Condon's (1987) map at $1.49 \mathrm{GHz}$, using the "best subtraction" method. This was done by subtracting at the given position the image of a point source with the flux density adjusted to reach a smoothly distributed emission in its environment. This procedure yielded some guess of the flux densities of subtracted sources at $4.85 \mathrm{GHz}$ which together with Condon's data allowed us to determine their spectral indices between these frequencies. All uncertainties of this procedure (determined by varying the $4.85 \mathrm{GHz}$ fluxes of subtracted sources) are included in the spectral index errors. The results are summarized in Table 2.

The brightest total power peak (Fig. 5) east of the optically bright galaxy is largely nonthermal. As it is partly polarized (Fig. 6) it is likely to be a background source unrelated to the galaxy, as already stated by Klein \& Gräve (1986). The brightness peaks associated with three nebulous objects in the northern galaxy region have mostly thermal spectra, north of the westernmost of them a nonthermal source was found (see Table 2). The east-west elongated feature in the southern region is also largely nonthermal, with $\alpha \simeq 0.6$ (computed between Condon's (1987) and our map).

In NGC 6822 we estimated the minimum content of the genuinely diffuse total (i.e. thermal and nonthermal) emission not clearly associated with any discrete features. This was done 


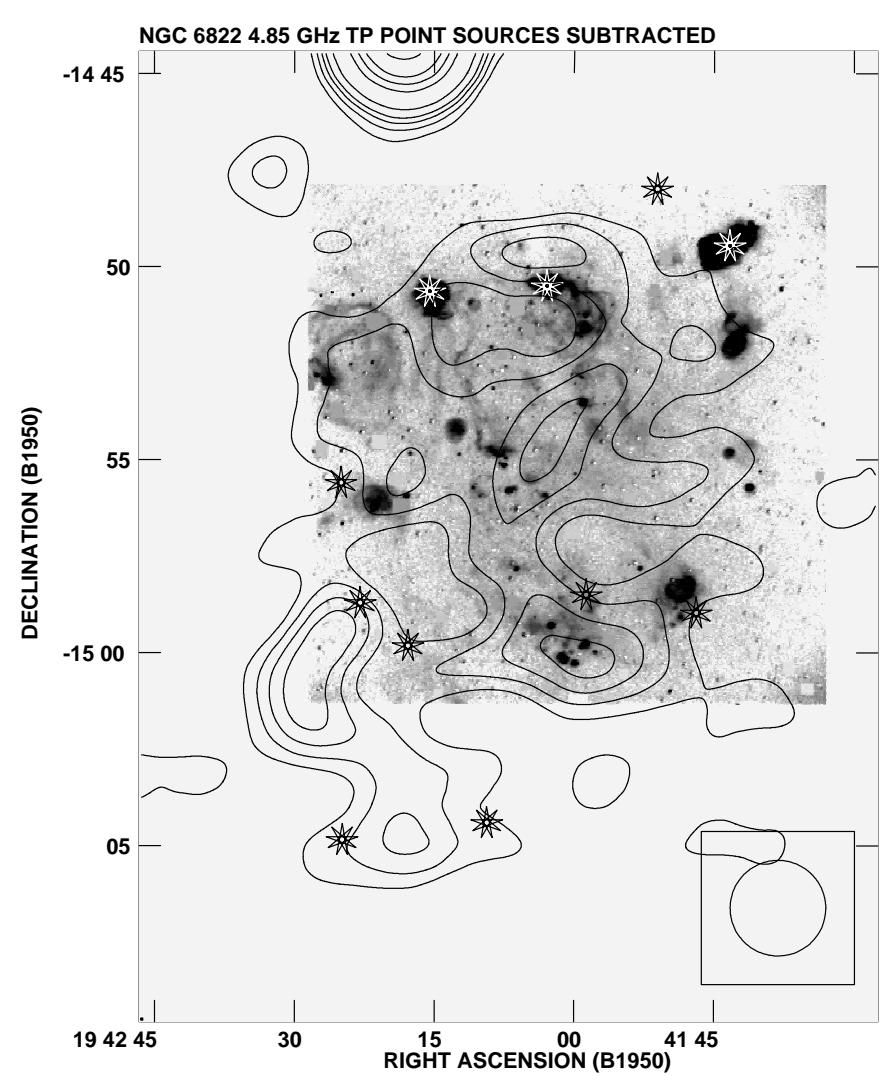

Fig. 9. The genuinely diffuse total power emission (both thermal and nonthermal) of NGC 6822 at $4.85 \mathrm{GHz}$ resulting from the "maximum subtraction experiment" (see text). The contour levels are 1, 2, 3, 4, 6 etc. $\mathrm{mJy} / \mathrm{b}$.a. The stars show the positions of subtracted point sources.

by performing the "maximum subtraction experiment" at the position of all sources from Condon's (1987) map. We subtracted the response of the Effelsberg telescope (taken from the calibration source map) with the maximum amplitude not producing negative responses (thus somewhat deeper than in the "best subtraction" used to determine the spectra in Table 2) allowing to estimate the emission unexplainable by discrete sources. We did the same at the positions of two groups of H II regions in the south-western region at $\mathrm{RA}_{1950}=19^{\mathrm{h}} 41^{\mathrm{m}} 58^{\mathrm{s}} .7$, $\operatorname{Dec}_{1950}=-14^{\circ} 58^{\prime} 30^{\prime \prime}$ and $\mathrm{RA}_{1950}=19^{\mathrm{h}} 41^{\mathrm{m}} 46^{\mathrm{s}} .9, \operatorname{Dec}_{1950}=$ $-14^{\circ} 58^{\prime} 58^{\prime \prime}$ (not seen in Condon's map), to be sure that all what remains is not associated with any discrete features. The result is shown in Fig. 9.

The maximum subtraction of all possible sources of localized emission has left a substantial diffuse component. Its integrated flux density amounts to $59 \pm 6 \mathrm{mJy}$, thus it constitutes some $40 \%$ of the total flux, the latter computed without the strong, dominant source east of the centre. This emission has no chance to be detected by the VLA at frequencies $\geq 1.4 \mathrm{GHz}$ because of its extent and low surface brightness. In addition to a weak diffuse component of the whole galaxy we note an extended region at $\mathrm{RA}_{1950}=19^{\mathrm{h}} 24^{\mathrm{m}} 27^{\mathrm{s}} \operatorname{Dec}_{1950}=$ $-15^{\circ} 00^{\prime}$ and its extension southwards to $\mathrm{RA}_{1950}=19^{\mathrm{h}} 42^{\mathrm{m}} 19^{\mathrm{s}}$ Dec $_{1950}=-15^{\circ} 05^{\prime}$. It is coincident with the western wall of an $\mathrm{H}$ I shell located east of the optical galaxy, discussed by de Blok \& Walter (2000). It extends southwards along the shell, while its mentioned southernmost peak coincides with the H I

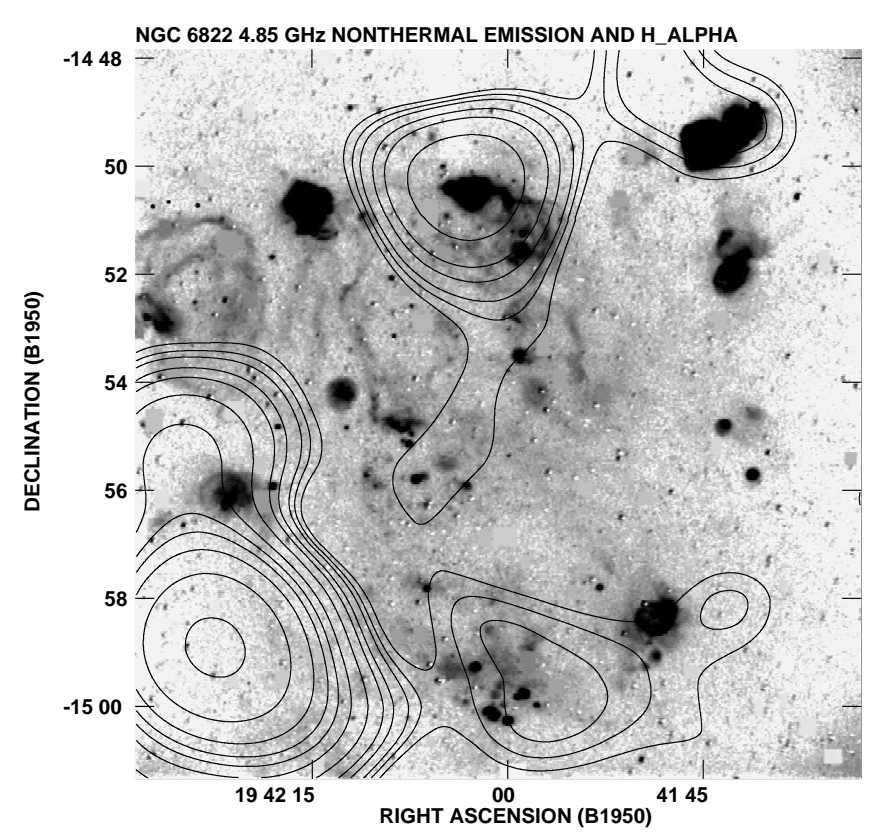

Fig. 10. The nonthermal emission at $4.85 \mathrm{GHz}$ from NGC 6822 estimated by subtracting thermal emission predicted from the $\mathrm{H} \alpha$ emission at $4.85 \mathrm{GHz}$ from our total power map. The extinction has been calibrated using the "best subtraction" radio and the $\mathrm{H} \alpha$ fluxes of three large H II regions in the northern region of NGC 6822. The contour levels are 1, 1.5, 2, 3, 4, 6, 9, 12, 15, 20, 30, 50 mJy/b.a.

maximum. The separation of this diffuse emission onto thermal and nonthermal components has to await a reliable determination of its spectrum and thermal content. This requires arcmin resolution observations of NGC 6822 at lower frequencies with an extreme sensitivity to large smooth structures.

An attempt to determine the distribution of total content of nonthermal emission (including the discrete sources) was made using the $\mathrm{H} \alpha$ map. We adjusted the extinction using the thermal sources revealed by the "best subtraction" method and the calibrated $\mathrm{H} \alpha$ fluxes of associated H II regions. NGC 6822 has a metallicity similar to that of IC 10 (Lisenfeld \& Ferrara 1998). Thus, as the bulk of extinction should occur in our Galaxy with little variations over the galaxy's body, we applied the same value to a whole map. The predicted distribution of thermal emission at $4.85 \mathrm{GHz}$ was subtracted from our total power map shown in Fig. 5. Results are shown in Fig. 10. The only definite nonthermal emission was found to come from the eastern strong source, from the region extending northwards of the $\mathrm{H}$ II complex at $\mathrm{RA}_{1950}=19^{\mathrm{h}} 41^{\mathrm{m}} 44^{\mathrm{s}} \operatorname{Dec}_{1950}=-19^{\circ} 49^{\prime} 30^{\prime \prime}$ (seen also in Condon's 1987 map at $1.49 \mathrm{GHz}$, and from the vicinity of star-forming region at $\mathrm{RA}_{1950}=19^{\mathrm{h}} 42^{\mathrm{m}} 02^{\mathrm{s}} \mathrm{Dec}_{1950}=$ $-19^{\circ} 50^{\prime} 30^{\prime \prime}$. For the latter we found a thermal spectrum, which signifies some nonthermal diffuse emission around the $\mathrm{H}$ II region, missing in Condon's (1987) map.

The evidence for the nonthermal emission from the inner galaxy regions depends strongly on the assumption of a constant extinction. The blob of nonthermal emission around $\mathrm{RA}_{1950}=19^{\mathrm{h}} 41^{\mathrm{m}} 56^{\mathrm{s}} \operatorname{Dec}_{1950}=-14^{\circ} 59^{\prime} 52^{\prime \prime}$ coincides with a dense H I cloud observed by de Blok \& Walter (2000) may be an artifact of an underestimated $\mathrm{H} \alpha$ flux, because of increased extinction. A nonthermal streak extending southwards from the 
$\mathrm{H}$ II region at $\mathrm{RA}_{1950}=19^{\mathrm{h}} 42^{\mathrm{m}} 02^{\mathrm{s}} \operatorname{Dec}_{1950}=-19^{\circ} 50^{\prime} 30^{\prime \prime}$ may disappear if extinction in the inner galaxy rises by some $50 \%$ compared to outer regions, as discussed by Massey et al. (1995). On the other hand it may get twice stronger if the rest of the galaxy shows less extinction than the northern star-forming regions by the same value. Even under these favorite conditions the equipartition strength of the total magnetic field is at most $5 \mu \mathrm{G}$ (assumptions like in Sect. 4.1.2), smaller than in the outer disk of IC 10 and very low with respect to spiral galaxies. The polarized emission in the northern region constitutes thus the only unambiguous evidence for diffuse nonthermal radiation from the inner part of NGC 6822, difficult to detect in total power because of 6.5 times higher noise.

We also computed the mean thermal fraction in NGC 6822 by integrating the total and thermal flux in identical areas delineated by the boundary of the $\mathrm{H} \alpha$ map. The thermal emission within this area amounts to some $45 \%$ with the bulk of the nonthermal flux coming from the region in the SE corner of the map shown in Fig. 10. Subtracting this region we find $\overline{f_{\text {th }}}$ at $4.85 \mathrm{GHz}$ in the inner galaxy's body of $75 \%$ changing between $50 \%$ and $90 \%$, depending whether the light from central part is by $50 \%$ more absorbed than in the outskirts (Massey et al. 1995) or whether there is more extinction in the northern $\mathrm{H}$ II regions for which we determined directly the absorption. Moreover, a large fraction of nonthermal emission comes from an isolated region around the mentioned $\mathrm{H}$ II region in the northern part of NGC 6822.

We estimate $\overline{f_{\text {th }}}$ at $10.45 \mathrm{GHz}>75 \%$, likely even as high as $80 \%-90 \%$, which is very high compared to spiral galaxies and considerably higher than in IC 10 . Most of the observed diffuse total power emission comes thus from the $\mathrm{H} \alpha$ structures filling the whole galaxy body. Week synchrotron emission with respect to the thermal emission was also found for NGC 5907 (Dumke et al. 2000).

\subsection{The magnetic field structure and origin}

The only significant polarization in IC 10 has been detected in the southern part of the galaxy, close to a straight dust feature and to a nonthermal blob detected there by Yang \& Skillman (1993). The equipartition regular field over the rest of IC 10 is much weaker than $3 \mu \mathrm{G}$. In contrast to optically larger irregulars almost all the polarization comes from an isolated region, while the detected regular magnetic field is parallel to the dust lane and is probably physically associated with local phenomena. The polarized nonthermal blob coincides with the brightest H I concentration in IC 10 (Wilcots \& Miller 1998). It lies at the southern boundary of one of large supershells blown by winds from star-forming regions. It also lies in the galaxy part where long H I extensions with peculiar velocities, possibly signifying gas accretion, are present. Though no direct coincidence of our polarized feature with these infalling gas streamers are found we speculate that it may be a combined result of the gas kinematics (compressions, shearing flows) driven by expanding superbubbles and gas infall. To distinguish between genuine regular magnetic fields and anisotropic, squeezed random ones, produced by "fluctuating dynamos"
(Subramanian 1998), then stretched and/or compressed by gas flows, needs information on the Faraday rotation, thus multifrequency polarization observations.

In NGC 6822 the bulk of polarized emission could be due to background sources in the eastern galaxy and south of the main stellar body (Fig. 6). On the other hand, we note that the eastern polarized region extends along the western wall of the H I shell located east of NGC 6822 (de Blok \& Walter 2000 ), with $\boldsymbol{B}$-vectors running almost parallel to the wall. The southern polarized feature seems to extend along the SW wall of the shell (as does its total power counterpart), again with $\boldsymbol{B}$ vectors running along the $\mathrm{H}$ I ridge. Moreover, it lies at the tip of the optical extension visible e.g. in Fig. 6. We cannot reject some association of these features with local compressional effects though the probability is rather low. High resolution observations are required to solve this problem.

Despite its weak synchrotron emission NGC 6822 also shows clear signs of diffuse polarized emission filling the northern part of its optical body. The $\boldsymbol{B}$-vectors in this area show some alignment with the $\mathrm{H} \alpha$ filaments (Fig. 5). The nonthermal radiation is so weak that the polarized signal falls between $2 \sigma$ and $3 \sigma$ rms noise hence the field direction has to be taken with extreme care. Nevertheless the polarization becomes significant after averaging it over large areas of several beams. Taking into account uncertainties of the thermal fraction, NGC 6822 shows on average 5-20\% nonthermal polarization, rising to $30-40 \%$ in the northern half of the galaxy. The regular field may amount to $2-3 \mu \mathrm{G}$ in this region.

Table 3 compares IC 10 and NGC 6822 to NGC 4449 - the irregular with much larger star-forming body (Klein et al. 1996; Chyży et al. 2000) and to the mean properties of spiral galaxies (Beck et al. 1996; Knapik et al. 2000). In all cases we refer to results obtained with single dish telescopes with a similar resolution relative to the galaxy size. Rapidly rotating spirals are dominated by synchrotron emission and their regular magnetic fields show typical characteristics of the classical $\alpha-\Omega$ dynamo process (see Beck et al. 1996; Knapik et al. 2000). The irregular galaxy NGC 4449 showing weaker signs of orderly rotation but having a quite large star-forming body ( $\simeq 9 \mathrm{kpc}$ in diameter) possesses still strong regular fields with characteristics of the dynamo process including its strong radial magnetic field (Chyży et al. 2000; Otmianowska-Mazur et al. 2000). Because of its slow rotation the classical dynamo process may be inefficient, but its modified version driven by Parker instabilities (Moss et al. 1999; Hanasz \& Lesch 1998) may still generate strong global magnetic fields. This galaxy has radio spectrum flatter than average for spiral galaxies, thus probably weaker synchrotron radiation (relative to its star-forming activity) and weaker CR electron confinement in large-scale magnetic fields. This may suggest a lower efficiency of the mechanism generating global fields.

In very rapidly star-forming IC 10 having a several times smaller optical (and ionized gas) body at least $60 \%$ of the flux at $10.45 \mathrm{GHz}$ is of thermal origin, moreover its flat nonthermal spectrum indicates an even smaller role of magnetic field in the CR confinement than in NGC 4449. This galaxy shows also much weaker global regular magnetic fields than the larger, similarly rotating NGC 4449. Either the mechanism producing 
Table 3. Magnetic fields in spiral and irregular galaxies.

\begin{tabular}{lccccl}
\hline \hline & $\begin{array}{c}<f_{\mathrm{nt}}> \\
10.45 \mathrm{GHz}\end{array}$ & $\begin{array}{c}<p> \\
\mathrm{nth}\end{array}$ & $\begin{array}{c}<B_{\mathrm{tot}}> \\
\mu \mathrm{G}\end{array}$ & $\begin{array}{c}<B_{\mathrm{reg}}> \\
\mu \mathrm{G}\end{array}$ & $\begin{array}{l}\text { gen. } \\
\text { reg. }\end{array}$ \\
\hline $\begin{array}{l}\text { Normal } \\
\text { spirals }\end{array}$ & $\leq 80 \%$ & $\simeq 5 \%$ & $5-15$ & $5-10$ & $\alpha-\Omega$ \\
\hline $\begin{array}{l}\text { Large irr. } \\
\text { High SF } \\
\text { (NGC 4449) }\end{array}$ & $\simeq 60 \%$ & $\simeq 5 \%$ & $5-15$ & $\simeq 5$ & $\alpha-\Omega^{*}$ \\
\hline $\begin{array}{l}\text { Small irr. } \\
\text { High SF }\end{array}$ & $\simeq 40 \%$ & $\left.<5 \%{ }^{1}\right)$ & $5-15$ & $\leq 3$ & $\begin{array}{l}\text { Fluct. dynamo+ } \\
\text { (IC 10) }\end{array}$ \\
\hline $\begin{array}{l}\text { Small irr. } \\
\text { low SF } \\
\text { (NGC 6822) }\end{array}$ & $\leq 25 \%$ & $\leq 20 \%$ & $\leq 5$ & $\leq 3$ & $\begin{array}{l}\text { Magn. field } \\
\text { diffusion? }\end{array}$ \\
\hline
\end{tabular}

\section{Abbreviations:}

gen./reg. - the generating mechanism of regular magnetic fields.

$\alpha-\Omega-$ the classical $\alpha-\Omega$ dynamo described by Ruzmaikin et al. (1988).

$\alpha-\Omega^{*}-$ the dynamo driven by magnetic instabilities (Moss et al. 1999; Hanasz \& Lesch 2000).

Fluct. dynamo - a "fluctuating dynamo" producing small scale magnetic fields (Subramanian 1998).

Magn. field diffusion - magnetic field produced in star-forming regions and diffusing into the rest of the galaxy.

Notes:

$\left.{ }^{1}\right)$ - average in the disk including compressed regions,

${ }^{2}$ ) - compressed regions excluded,

${ }^{3}$ ) - generation of random fields by the "fluctuating dynamo" followed by their compression and stretching in gas flows. They will be then anisotropic random fields rather than genuine unidirectional magnetic fields.

large-scale magnetic fields is much less efficient because of its smaller size, or a strong concentration of star formation in a small volume destroys regular fields efficiently. An apparent lack of a magnetized halo, usually produced by the dynamo process, favours the first hypothesis namely a galaxy whose size is below the threshold of large-scale mean-field dynamo. However, random fields may be still efficiently produced by local "fluctuating dynamos" (Subramanian 1998), producing small-scale magnetic fields due to turbulent gas motions.

The production of a random magnetic field is even weaker (relative to the star formation level) in NGC 6822, the synchrotron emission fades quickly with increasing distance from the star-forming nests. This may mean too low an energy input from star-forming processes for the "fluctuating dynamo". We note some evidence for regular fields in the northern part of NGC 6822. It is unlikely that this is a relic field produced during a past star formation burst, as NGC 6822 had been quietly and slowly forming stars during past 10 Gyr (Wyder 2001). We may speculate that some magnetic field is still (or has been recently) produced by the "fluctuating dynamo" in the northern star-forming regions (note the diffuse nonthermal emission around one of them), then diffusing into the galaxy body, getting gradually more regular as the turbulence decays (Antonov et al. 2000).

\subsection{The ionized gas}

We discovered $\mathrm{H} \alpha$-emitting features not obviously associated with star-forming regions in both galaxies. This finding is surprising in NGC 6822 in which diffuse $\mathrm{H} \alpha$ emission is found to fill the whole galaxy's body, given the very few sources of ionising radiation.

Using our estimates of thermal and nonthermal emission we compared the ratio of thermal and magnetic energy density $\left(E_{\mathrm{th}} / E_{\mathrm{B}}\right)$ in IC 10 and in the normal spiral galaxy NGC 6946 (Ehle \& Beck 1993). In the latter case, for the ionized gas properties (electron temperature, volume filling factor) similar to those in Ehle \& Beck (1993) $E_{\text {th }} / E_{\mathrm{B}}$ was found to be between 1 and 1.5, depending on the galactocentric radius and the exact values of the ISM parameters. This means the energy equipartition between the magnetic field and ionized gas. Under the same assumptions we obtained for IC 10 values of $E_{\mathrm{th}} / E_{\mathrm{B}}$ ranging from 1.5-2 for a thermal fraction of 0.6 and a thermal gas and synchrotron emission line of sight thickness of $100 \mathrm{pc}$ and $1 \mathrm{kpc}$ respectively. However, $E_{\mathrm{th}} / E_{\mathrm{B}}$ becomes similar to the values for NGC 6946 if either a thicker ionized gas layer $(200 \mathrm{pc})$ or thinner synchrotron disk $(800 \mathrm{pc}$, both within uncertainties) is adopted. Thus in IC 10 magnetic fields might play a significant role in local gas dynamics (like the cloud evolution, triggering star formation via MHD instabilities) as it does in large irregulars or in large spirals.

A long filament extending to the SW and connected to H II regions inside IC 10 seems to have comparable magnetic and thermal energy densities (assuming a diffuse, smooth magnetic field around the filament). The magnetic energy may be dominant if we assume the magnetic field to be concentrated in the filament, as indeed suggested by a rather narrow ridge of nonthermal emission. Magnetic forces can be important in the 
dynamics of this feature, but this conclusion strongly depends on the filling factor of magnetic field. Its knowledge needs observations with much higher resolution.

The above is not true in the northern star-forming complex, being completely dominated by the thermal gas. The same is valid for a detached diffuse $\mathrm{H} \alpha$ emitting nebulosity west of this star-forming region. Magnetic forces seem to play only a minor role there.

\section{Summary and conclusions}

We performed a sensitive search for the extended total power and polarized radio emission from two irregular galaxies: the blue compact dwarf IC 10 and the slowly star forming NGC 6822, accompanied by sensitive mapping of the ionized gas. The galaxies rotate slowly and are small, making the dynamo action very difficult. The following results were obtained:

- IC 10 shows two isolated strongly star-forming nests and was found to possess a nonthermal radio envelope. The thermal fraction is much larger than in normal spirals. The galaxy shows strong but largely random magnetic field with a strength of $14 \pm 4 \mu \mathrm{G}$. The nonthermal emission (signifying widespread magnetic fields) constitutes a smaller fraction of the total flux at $10.45 \mathrm{GHz}$ than in spirals and in large irregulars. At least $60 \%$ of emission at this frequency is likely to be thermal.

- NGC 6822 shows a much lower level of recent star formation and still possesses some weak extended radio continuum emission, partly of synchrotron nature which is difficult to prove because of lack of reliable data at other frequencies. Using the Mt. Laguna $\mathrm{H} \alpha$ data and subtracting the bright nonthermal (probably background) source we find that more than $80-90 \%$ of its emission (scaled to $10.45 \mathrm{GHz}$ ) may be thermal. The total magnetic field ( $\leq 5 \mu \mathrm{G}$ in the inner body) is very weak compared to large irregular and spiral galaxies.

- Both galaxies were found to possess a system of $\mathrm{H} \alpha$ filaments present also outside star-forming regions. In IC 10 the ionized gas filament extending by $0.68 \mathrm{kpc}$ from the optically bright galaxy is associated with a similar synchrotron feature, suggesting a dynamically important magnetic field. Another, more clumpy ionized gas cloud is dominated by thermal processes.

- Both galaxies show localized traces of polarized emission indicating the presence of regular magnetic fields of strength of $2-3 \mu \mathrm{G}$. In IC 10 it is clearly associated with a dust lane and a nonthermal bubble and is probably due to compression effects. In NGC 6822 it is much less clear, however, there is a weak and still disputable indication of some regular field associated with a large H I shell. We detected also clear traces of diffuse polarized emission in the northern region of the galaxy.

- Rapidly star-forming IC 10 lacks clear signs of the global regular fields as expected for the classical mean-field dynamo. An efficient mechanism generating purely random field (e.g. the "fluctuating dynamo", Subramanian 1998) is likely to work. Nonthermal polarization seems to be considerably higher (locally up to $30-40 \%$ ) in the more quiet NGC 6822. We speculate that the magnetic field may be generated in scarce star-forming regions (e.g. via the "fluctuating dynamo"), diffusing into quiet regions devoid of current star formation.

We found that the rapid rotation is not the only critical agent in generating strong total magnetic fields filling smoothly the whole galaxy. However, in small objects no strong regular fields are produced though they can arise locally due to local compressions. In case of very weak star formation the synchrotron emission is extremely weak compared to the thermal one. Nevertheless, even in this case local regular fields can be found. Multifrequency radio observations with a very good sensitivity to extended structures are highly desirable.

Acknowledgements. The Authors wish to express their thanks to the colleagues from the Max-Planck-Institut für Radioastronomie (MPIfR) in Bonn for their valuable discussions during this work. J.K., M.S., K.Ch. and M.U. are indebted to Professor Richard Wielebinski from the MPIfR for the invitations to stay at this Institute, where substantial parts of this work were done. M.S. K.Ch. and M.U. are also indebted to Professor Ralf-Jürgen Dettmar from Ruhr-Universität Bochum for arranging the working visits at this University. The Authors are also grateful to Dr Marita Krause for careful reading of the manuscript. This work was supported by a grant from the Polish Research Committee (KBN), grants no. 962/P03/97/12 and 4264/P03/99/17. DJB thanks the DFG for support of the Calar observing run, H. Domgörgen for participating in the October 1994 observing run, and the Humboldt Foundation for their support from a Feodor-Lynen Fellowship during which the observations at Mt. Laguna were taken. DJB thanks the director and the staff of the Astronomical Observatory of the Jagiellonian University for their hospitality during his visits. We have made use of the LEDA database (http://leda.univ-lyon1.fr).

\section{References}

Antonov, T., Frick, P., \& Sokoloff, D. 2000, Num. Methods and Programming, 1, 14

Baars, J. W. M., Genzel, R., Pauliny-Toth, I. I. K., \& Witzel, A. 1977, A\&A, 61, 99

Beck, R., Brandenburg, A., Moss, D., Shukurov, A., \& Sokoloff, D. 1996, ARA\&A, 34, 155

Beck, R., \& Hoernes, P. 1996, Nature, 379, 47

Bomans, D. J., Chu, Y. H., \& Hopp, U. 1997, AJ, 113, 1678

Blitz, L., \& Shu, F. M. 1980, ApJ, 138, 148

Chyży, K. T., Beck, R., Kohle, S., Klein, U., \& Urbanik, M. 2000, A\&A, 356, 757

Chyży, K. T. 2002, Highlights Astron., 12, 721

Condon, J. J. 1987, ApJS, 65, 485

de Blok, W. J. G., \& Walter, F. 2000, ApJ, 537, L95

Dettmar, R.-J. 1993, Rev. Mod. Astron., 6, 33

Dumke, M., Krause, M., \& Wielebinski, R. 2000, A\&A, 355, 512

Ehle, M., \& Beck, R. 1993, A\&A, 273, 45

Emerson, D. T., Klein, U., \& Haslam, C. G. T. 1979, A\&A, 76, 92

Emerson, D. T., \& Gräve, R. 1988, A\&A, 190, 353

Ferriere, K. M., Mac Low, M.-M., \& Zweibel, E. G. 1991, ApJ, 375, 239

Hanasz, M., \& Lesch, H. 1998, A\&A, 332, 77

Hanasz, M., \& Lesch, H. 2000, ApJ, 543, 235

Haslam, C. G. T. 1974, A\&AS, 15, 333 
Hodge, P., Kennicutt, R. C., \& Lee, M. G. 1988, PASP, 100, 917

Hodge, P., \& Lee, M. G. 1990, PASP, 102, 26

Huchtmeier, W. K. 1979, A\&A, 75, 170

Hunter, D. A., \& Gallagher, J. S. 1990, ApJ, 362, 480

Hunter, D. A., Hawley, W. N., \& Gallagher, J. S. 1993, AJ, 106, 1797

Hunter, D. A. 2000, A\&AS, 197, 7906

Klein, U., Gräve, R., \& Wielebinski, R. 1983, A\&A, 117, 332

Klein, U., \& Gräve, R. 1986, A\&A, 161, 155

Klein, U., Weiland, H., \& Brinks, E. 1991, A\&A, 246, 323

Klein, U., Haynes, R. F., Wielebinski, R., \& Meinert, D. 1993, A\&A, 271,402

Klein, U., Hummel, E., Bomans, D. J., \& Hopp, U. 1996, A\&A, 313, 396

Knapik, J., Soida, M., Beck, R., Dettmar, R.-J., \& Urbanik, M. 2000, A\&A, 362, 910

Lisenfeld, U., \& Ferrara, A. 1998, ApJ, 496, 145

Lo, K. Y., Sargent, W. L. W., \& Young, K. 1993, AJ, 106, 507

Lequeux, J., Rayo, J. F., Serrano, A., Peimbert, M., \& Torres-Peimbert, S. 1979, A\&A, 80, 155

Mac Low, M.-M., \& Ferrara, A. 1998, Lecture Notes Phys., 506, 559

Massey, P., Armandroff, T. E., Pyke, R., Patel, K., \& Wilson, C. D. 1995, AJ, 110, 2715

Mathis, J. S. 1979, ApJ, 232, 747

McGonegal, R., McLaren, R. A., Welch, D. L., Madore, B. F., \& McAlary, C. W. 1983, ApJ, 273, 539

Morsi, H. W., \& Reich, W. 1986, A\&A, 163, 313
Moss, D., Shukurov, A., \& Sokoloff, D. 1999, A\&A, 343, 120

Niklas, S., Klein, U., \& Wielebinski, R. 1997, A\&A, 322, 19

Otmianowska-Mazur, K., Chyży, K. T., Soida, M., \& von Linden, S. 2000, A\&A, 359, 29

Pacholczyk, A. G. 1970, Radio Astrophysics (S. Francisco: Freeman) Rengelink, R. B., Tang, Y., de Bruyn, A. G., et al. 1997, A\&AS, 124, 259

Richer, M. G., Bullejos, A., Borissova, J., et al. 2001, A\&A, 370, 34

Ruzmaikin, A. A., Shukurov, A. M., \& Sokoloff, D. D. 1988, Magnetic Fields of Galaxies, Astrophys. and Space Science Library, Vol. 133 (Kluwer Academic Publishers)

Sabbadin, F., \& Bianchini, A. 1979, PASP, 91, 280

Schlegel, D. J., Finkbeiner, D. P., \& Davis, M. 1998, ApJ, 500, 525

Schmidt, A., Wongsowijoto, A., Lochner, O., et al. 1993, MPIfR Technical Report No. 73, MPIfR, Bonn

Shostak, G. S. 1974, A\&A, 31, 97

Simard-Normandin, M., \& Kronberg, P. P. 1980, ApJ, 242, 74

Soida, M., Urbanik, M., \& Beck, R. 1996, A\&A, 312, 409

Subramanian, K. 1998, MNRAS, 294, 718

Tabara, H., \& Inoue, M. 1980, A\&AS, 39, 379

White, R. L., \& Becker, R. H. 1992, ApJS, 79, 331

Wilcots, E. M., \& Miller, B. W. 1998, AJ, 116, 2363

Wilson, C. D., Welch, D. L., Reid, I. N., Saha, A., \& Hoessel, J. 1996, AJ, 111, 1106

Wyder, T. K. 2001, AJ, 122, 2490

Yang, H., \& Skillman, E. D. 1993, AJ, 106, 1448 